\title{
State Reconstruction for Complex Dynamical Networks with Noises
}

\author{
Chunxia Fan, Guoping Jiang \\ College of Automation, Nanjing University of Posts and Telecommunications, Nanjing, China \\ Email: fancx@njupt.edu.cn
}

Received January 30, 2012; revised February 25, 2012; accepted March 12, 2012

\begin{abstract}
The state reconstruction problem is addressed for complex dynamical networks coupled with states and outputs respectively, in a noisy transmission channel. By using Lyapunov stability theory and $H_{\infty}$ performance, two schemes of state reconstruction are proposed for the complex dynamical networks with the nodes coupled by states and outputs respectively, and the estimation errors are convergent to zeros with $H_{\infty}$ performance index. A numerical simulation demonstrates the effectiveness of the proposed observers.
\end{abstract}

Keywords: State Reconstruction; Complex Dynamical Networks; Noisy Circumstance; $H_{\infty}$ Performance

\section{Introduction}

Complex dynamical networks have recently been a hot topic in science and engineering fields because it can describe many phenomena in nature and engineering [1-4]. For instance, power grid is a complex network if the electrical equipments are treated as nodes and the interactions between the equipments as edges; the individuals are treated as nodes and the interactions between the individuals as edges in a community then the community can be described as a network.

The synchronization of a complex dynamical network has been reported in the latest decade including inner synchronization and outer synchronization [5-12]. All state variables are required to construct the synchronization controllers in $[5-7,11]$. In $[8,10,12]$, partial state variables are needed to construct the synchronization controllers. For outer synchronization, the circumstance noise is considered in [9].

Recently, topology identification, fault diagnosis and parameter identification [13-20] of complex networks have become hot topic in complex network applications, and network synchronization has found applications in these fields. For topology identification, it is assumed that all of the states are available for a complex network in $[15,17-20]$. For monitoring topology change of network, it is assumed that the partial state variables are required for the network in $[14,16]$.

In the above study and other fields of complex net-

*This work was supported in part by the National Natural Science Foundation of China under 60874091 and 61104103 , the Natural Science Foundation for Colleges and Universities in Jiangsu Province, China under 10KJB120001. work, all or partial state variables are needed for design. However, for a large scale network, measuring all state variables is not easy or even impossible in practice, and locating many sensors costs much. Therefore, it is very important to estimate or reconstruct all state variables based on some limited available network information. For discrete complex networks, state reconstruction has been reported in [21]. For continuous time complex dynamical networks with transmission noise, there has been little theoretical work on state estimation in the literature.

Motivated by the above observations, in this paper we study the state reconstruction or state estimation problem for a complex network with transmission channel noise. By using Lyapunov stability theory and $H_{\infty}$ control theory, some state reconstruction schemes are derived for complex dynamical networks including state coupling and outputs coupling. For suppressing noise in the channel, the integral observers [22,23] are applied and the estimation errors are bounded with $H_{\infty}$ performance. Some numerical examples are given to shown the effecttiveness of the proposed schemes.

The rest of this paper is organized as follows. In Section 2 , the state reconstruction of state coupling networks is studied and some estimation criteria are derived in the form of linear matrix inequality. In Section 3, the state reconstruction of output coupling networks is studied and some estimation criteria are given. Some examples are given in Section 4 and conclusions are drawn in Section 5.

\section{State Reconstruction of Networks Coupled with States}

Consider a general complex dynamical network consist- 
ing of $N$ identical nodes with states couplings, which is described as follows

$$
\dot{x}_{i}=A x_{i}+f\left(x_{i}\right)+\sum_{j=1}^{N} c_{i j} \Gamma x_{j} \quad y_{i}=H x_{i}
$$

where $1 \leq i \leq N, x_{i}=\left(x_{i 1}, x_{i 2}, \cdots, x_{i n}\right)^{T} \in R^{n}$ is the state vector of the $i^{\text {th }}$ node, $y_{i} \in R^{m}$ is the output vector of the $i^{\text {th }}$ node, $A \in R^{n \times n}$ is the system matrix of node $i$, $f: R^{n} \rightarrow R^{n}$ is a nonlinear smooth vector field, $H \in R^{m \times n}$ is the output matrix of node $i, \Gamma \in R^{n \times n}$ is the coupling matrix of node $i$, node dynamics is $\dot{x}=A x+f(x)$, $C=\left(c_{i j}\right)_{N \times N} \in R^{N \times N}$ is the coupling configuration matrix. If there is a link from node $i$ to node $j(i \neq j)$, then $c_{i j}=1$; otherwise $c_{i j}=0$. Assume that $C$ is a diffusive matrix satisfying $\sum_{j=1}^{N} c_{j \neq i}=-c_{i i}$. It is noted that the configuration matrix $C$ does not need to be irreducible and symmetric.

Hypothesis 1: (H1) Suppose that $f(x)$ is Lipschitz continuous. That is, there exists a positive number constant $\alpha$ satisfying $\|f(\hat{x})-f(x)\| \leq \alpha\|\hat{x}-x\|$ for $x \in R^{n}$, $\hat{x} \in R^{n}$, where \|\| represents the Euclidean normal.

For most networks, all of the states are generally not available. To reconstruct the states of network (1), outputs $y_{i}$ are transmitted from (1) to the observer through the transmission channel. In the practical engineering, there exists noise in the transmission channel. Therefore, the measurements received by the observer are characterized by

$$
\bar{y}_{i}=H x_{i}+w_{i} \quad(i=1,2, \cdots, N)
$$

where $\bar{y}_{i} \in R^{m}$ is the actual measurement outputs and $w_{i}$ are the noises in the transmission channel.

Hypothesis 2: (H2) Suppose that the disturbances in the transmission channel are bounded, i.e., there exists a positive constant $d$ such that $\left\|w_{i}\right\| \leq d$.

To reconstruct all the states of (1), the following observer is presented

$$
\begin{aligned}
& \dot{\hat{x}}_{i}=A \hat{x}_{i}+f\left(\hat{x}_{i}\right)+\sum_{j=1}^{N} c_{i j} \Gamma \hat{x}_{j}+\bar{K}_{i}\left(z_{i}-\hat{z}_{i}\right) \\
& \dot{z}_{i}=\bar{y}_{i}=H x_{i}+w_{i} \quad \dot{\hat{z}}=\hat{y}_{i}=H \hat{x}_{i}+l_{i}\left(z_{i}-\hat{z}_{i}\right)
\end{aligned}
$$

where $i=1,2, \cdots, N, \hat{x}_{i} \in R^{n}$ is the state estimated for $i^{\text {th }}$ node in the network (1), $\hat{y}_{i} \in R^{m}$ is the output vector of the $i^{\text {th }}$ in (3), $\bar{K}_{i} \in R^{n \times m}$ and $l_{i} \in R^{m \times m}$ are the observer gain matrices to be determined.

Remark 1: The observer (3) is different from the traditional proportional observer because its controller is the integral of the measurements [22,23]. The states reconstructed by (3) can better converge to the states of (1) since the disturbance is not amplified if a large proportional gain $\bar{K}_{i}$ is used [8].

The aim is to determine appropriate observer gain matrices $\bar{K}_{i} \in R^{n \times m}$ and $l_{i} \in R^{m \times m}$ such that the reconstructed states $\hat{x}_{i}$ approach the network states $x_{i}$.

Define the state errors

$$
\begin{aligned}
& e_{i}=x_{i}-\hat{x}_{i} \\
& e_{z i}=z_{i}-\hat{z}_{i}
\end{aligned} \quad i=1,2, \cdots, N
$$

Then it follows from (1) and (3) that

$$
\begin{aligned}
& \dot{e}_{i}=A e_{i}+f\left(x_{i}\right)-f\left(\hat{x}_{i}\right)+\sum_{j=1}^{N} c_{i j} \Gamma e_{j}-\bar{K}_{i} e_{z i} \\
& \dot{e}_{z i}=H e_{i}+w_{i}-l_{i} e_{z i} \quad i=1,2, \cdots, N
\end{aligned}
$$

Define $B=\left[\begin{array}{ll}A & 0 \\ H & 0\end{array}\right], G=\left[\begin{array}{ll}0 & \Gamma \\ 0 & 0\end{array}\right], K_{i}=\left[\begin{array}{c}\bar{K}_{i} \\ l_{i}\end{array}\right]$,

$E_{i}=\left[\begin{array}{ll}e_{i}^{T} & e_{z i}^{T}\end{array}\right]^{T}, F\left(x_{i}, \hat{x}_{i}\right)=\left[\begin{array}{ll}\left(f\left(x_{i}\right)-f\left(\hat{x}_{i}\right)\right)^{T} & 0\end{array}\right]^{T}$,

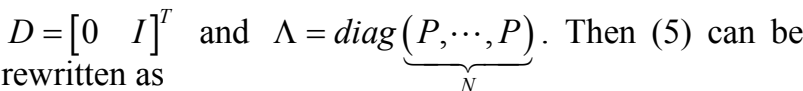

$$
\dot{E}_{i}=B E_{i}+F\left(x_{i}, \hat{x}_{i}\right)+\sum_{j=1}^{N} c_{i j} \Gamma G E_{j}+D w_{i}-K_{i} D^{T} E_{i}
$$

Then we will derive $K_{i}$ to guarantee that $E_{i}$ are convergent to zeros when $w_{i}=0$, and $E_{i}$ are convergent to zeros with $H_{\infty}$ performance $\gamma$, characterized by the following inequality, when $w_{i} \neq 0$ and $\left\|w_{i}\right\| \leq d$.

$$
\int_{0}^{+\infty}\left\|E_{i}(t)\right\|^{2} \mathrm{~d} t \leq \gamma^{2} \int_{0}^{+\infty}\left\|w_{i}(t)\right\|^{2} \mathrm{~d} t \quad E_{i}(0)=0
$$

Theorem 1: Suppose that $\mathrm{H} 1$ and $\mathrm{H} 2$ hold. If there exist matrices $P=P^{T}>0, M_{i}(i=1,2, \cdots, N)$ and a constant $\gamma>0$ such that the following inequality holds

$$
\Pi+C \otimes(P \Gamma)+(C \otimes(P \Gamma))^{T}<0
$$

where $\Pi=\operatorname{diag}\left(P B+B^{T} P+\alpha^{2} I+P\left(I+D D^{T}\right) P+\gamma^{-2} I\right.$ $-D M_{1}^{T}-M_{1} D^{T}, \cdots, P B+B^{T} P+\alpha^{2} I+P\left(I+D D^{T}\right) P+\gamma^{-2} I$ $\left.-D M_{N}^{T}-M_{N} D^{T}\right)$, then the error dynamical system (6) will converge to zeros with $H_{\infty}$ performance $\gamma$ when $w_{i} \neq 0$. Consequently, network (3) can estimate the state of network (1) with $H_{\infty}$ performance $\gamma$ when $w_{i} \neq 0$ and $K_{i}=P^{-1} M_{i}$.

Proof: Define a Lyapunov function $V=\sum_{i=1}^{N} E_{i}^{T} P E_{i}$. Differentiating $V$ along the error dynamical system (6) and using Hypothesis 1, one obtains

$$
\begin{aligned}
\dot{V} & =\sum_{i=1}^{N}\left(E_{i}^{T}\left(P B+B^{T} P-D K_{i}^{T} P-P K_{i} D^{T}\right) E_{i}+2 E_{i}^{T} P F\left(x_{i}, \hat{x}_{i}\right)+E_{i}^{T} P \sum_{j=1}^{N} c_{i j} \Gamma G E_{j}+\left(\sum_{j=1}^{N} c_{i j} \Gamma G E_{j}\right)^{T} P E_{i}+2 E_{i}^{T} P D w_{i}\right) \\
& \leq \sum_{i=1}^{N}\left(E_{i}^{T}\left(P B+B^{T} P-D K_{i}^{T} P-P K_{i} D^{T}+\alpha^{2} I+P\left(I+D D^{T}\right) P\right) E_{i}+w_{i}^{T} w_{i}+E_{i}^{T} P \sum_{j=1}^{N} c_{i j} \Gamma G E_{j}+\left(\sum_{j=1}^{N} c_{i j} \Gamma G E_{j}\right)^{T} P E_{i}\right)
\end{aligned}
$$


Define $E=\left(E_{1}^{T}, \cdots, E_{N}^{T}\right)^{T}$. Using (8), one obtains

$$
\begin{aligned}
\dot{V} & \leq E^{T}\left(\Pi+C \otimes(P \Gamma)+(C \otimes(P \Gamma))^{T}\right) E \\
& -\sum_{i=1}^{N}\left(\gamma^{-2} E_{i}^{T} E_{i}-w_{i}^{T} w_{i}\right) \\
& \leq E^{T}\left(\Pi+C \otimes(P \Gamma)+(C \otimes(P \Gamma))^{T}\right) E \\
& -\sum_{i=1}^{N}\left(\gamma^{-2} E_{i}^{T} E_{i}-w_{i}^{T} w_{i}\right)
\end{aligned}
$$

From (10) and Lyapunov stability theorem, $E_{i}$ are exponentially convergent to zeros when $w_{i}=0$. Under $E_{i}(0)=0$, integrating (10) from 0 to $\infty$ yields that $H_{\infty}$ performance $\gamma$. The proof is completed.

To easily solve the matrix inequality, Schur complements lemma [9] is used here. Then (8) is transformed into the following linear matrix inequality

$$
\left[\begin{array}{cc}
\Delta+C \otimes(P \Gamma)+(C \otimes(P \Gamma))^{T} & \Lambda \\
\Lambda & -\left(I+D D^{T}\right)^{-1}
\end{array}\right]<0(10)
$$

where $\Delta=\operatorname{diag}\left(P B+B^{T} P+\alpha^{2} I+\gamma^{-2} I-D M_{1}^{T}-M_{1} D^{T}\right.$, $\left.\cdots, P B+B^{T} P+\alpha^{2} I+\gamma^{-2} I-D M_{N}^{T}-M_{N} D^{T}\right)$, and $\Lambda=\operatorname{diag}(P, \cdots, P)$.

\section{State Reconstruction of Networks Coupled with Outputs}

Next, we consider a complex dynamical network consisting $\mathrm{N}$ identical nodes coupled with the outputs characterized by

$$
\dot{x}_{i}=A x_{i}+f\left(x_{i}\right)+\sum_{j=1}^{N} c_{i j} L y_{j} \quad y_{i}=H x_{i}
$$

where $L \in R^{n \times m}$.

The outputs $y_{i}$ are disturbed by noise when they are transmitted from network (12) to the observer. Therefore information received by the observer is characterized by (2). The observer is designed as the following form

$$
\begin{aligned}
& \dot{\hat{x}}=A \hat{x}_{i}+f\left(\hat{x}_{i}\right)+\sum_{j=1}^{N} c_{i j} L \hat{y}_{j}+\bar{K}_{i}\left(z_{i}-\hat{z}_{i}\right) \\
& \dot{z}_{i}=\bar{y}_{i}=H x_{i}+w_{i} \quad \dot{\hat{z}}=\hat{y}_{i}=H \hat{x}_{i}+l_{i}\left(z_{i}-\hat{z}_{i}\right)
\end{aligned}
$$

where $\bar{K}_{i} \in R^{n \times m}$ and $l_{i} \in R^{m \times m}$ are the observer gain matrices to be determined. Then one can obtain the error dynamics

$$
\begin{aligned}
& \dot{e}_{i}=A e_{i}+f\left(x_{i}\right)-f\left(\hat{x}_{i}\right)+\sum_{j=1}^{N} c_{i j} L H e_{j}-\bar{K}_{i} e_{z i} \quad i=1,2, \cdots, N \\
& \dot{e}_{z i}=H e_{i}+w_{i}-l_{i} e_{z i}
\end{aligned}
$$

Let $E_{i}=\left[\begin{array}{ll}e_{i}^{T} & e_{z i}^{T}\end{array}\right]^{T}$, then (14) can be rewritten as

$$
\dot{E}_{i}=B E_{i}+F\left(x_{i}, \hat{x}_{i}\right)+\sum_{j=1}^{N} c_{i j} \bar{L} \bar{H} E_{j}+D w_{i}-K_{i} D^{T} E_{i}
$$

where $\bar{L}=\left[\begin{array}{ll}0 & L \\ 0 & 0\end{array}\right]$ and $\bar{H}=\left[\begin{array}{ll}H & 0\end{array}\right]$. Consequently, determine matrices $K_{i}$ such that error dynamics (15) is stable with $H_{\infty}$ performance, that is, observer (13) reconstruct the states of network (12) with $H_{\infty}$ performance. The following theorem gives the criteria of determining matrices $K_{i}$.

Theorem 2: Suppose that $\mathrm{H} 1$ and $\mathrm{H} 2$ hold. If there exist matrices $P=P^{T}>0, M_{i}(i=1,2, \cdots, N)$ and a constant $\gamma>0$ such that the following inequality holds

$$
\Pi+C \otimes(P \bar{L} \bar{H})+(C \otimes(P \bar{L} \bar{H}))^{T}<0
$$

where $\Pi=\operatorname{diag}\left(P B+B^{T} P+\alpha^{2} I+P\left(I+D D^{T}\right) P+\gamma^{-2} I\right.$ $-D M_{1}^{T}-M_{1} D^{T}, \cdots, P B+B^{T} P+\alpha^{2} I+P\left(I+D D^{T}\right) P+\gamma^{-2} I$ $\left.-D M_{N}^{T}-M_{N} D^{T}\right)$ then the error dynamical system (15) will converge to the zeros with $H_{\infty}$ performance $\gamma$ when $w_{i} \neq 0$. Consequently, observer (13) can estimate the state of network (12) with $H_{\infty}$ performance $\gamma$ when $w_{i} \neq 0$ and $K_{i}=P^{-1} M_{i}$.

The proof of Theorem 2 is similar to that of Theorem 1 , so it is omitted here.

Using Schur complement lemma, one obtains the following linear matrix inequality

$$
\left[\begin{array}{cc}
\Delta+C \otimes(P \bar{L} \bar{H})+(C \otimes(P \bar{L} \bar{H}))^{T} & \Lambda \\
\Lambda & -\left(I+D D^{T}\right)^{-1}
\end{array}\right]<0
$$

where $\Delta=\operatorname{diag}\left(P B+B^{T} P+\alpha^{2} I+\gamma^{-2} I-D M_{1}^{T}-M_{1} D^{T}\right.$, $\left.\cdots, P B+B^{T} P+\alpha^{2} I+\gamma^{-2} I-D M_{N}^{T}-M_{N} D^{T}\right)$ and $\Lambda=\operatorname{diag}(P, \cdots, P)$.

Remark 2: In this section, the complex dynamical network coupled with the outputs is considered because this kind of networks is practical in engineering for saving communications and sensors. When the transmission channel is ideal, the observer (13) can reconstruct the states of network (12) with exponential convergence. When the transmission channel is noisy, the observer can reconstruct the states of network (12) with $H_{\infty}$ performance.

\section{Numerical Simulations}

In this section, some numerical examples are given to demonstrate the effectiveness of the proposed state reconstructtion scheme for complex dynamical networks. In the network, chaotic Lorenz system is taken as the node dynamics.

Lorenz chaotic system is a well-known typical benchmark chaos, which can be described by the following [10] 

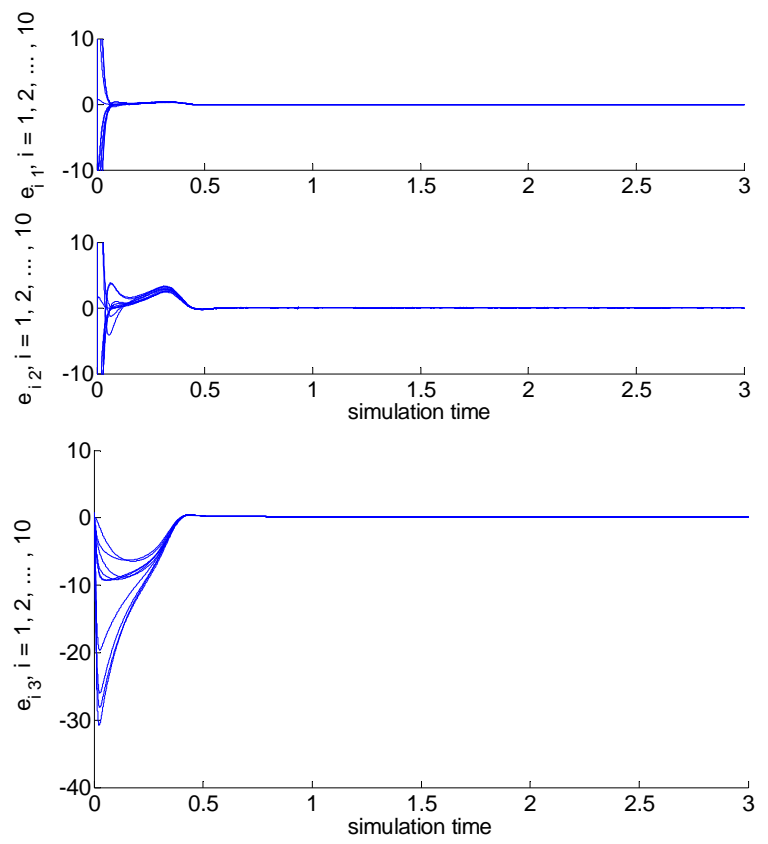

Figure 1. Errors between small world network (1) and observer (3).

$$
\begin{aligned}
\left(\begin{array}{l}
\dot{x}_{i 1} \\
\dot{x}_{i 2} \\
\dot{x}_{i 3}
\end{array}\right) & =\left(\begin{array}{ccc}
-a & a & 0 \\
b & -1 & 0 \\
0 & 0 & c
\end{array}\right)\left(\begin{array}{l}
x_{i 1} \\
x_{i 2} \\
x_{i 3}
\end{array}\right)+\left(\begin{array}{l}
0 \\
-x_{i 1} x_{i 3} \\
x_{i 1} x_{i 2}
\end{array}\right) \\
& =A x_{i}+f\left(x_{i}\right)
\end{aligned}
$$

where $a, b$ and $c$ are parameters. When $a=10, b=28$ and $c=-8 / 3$, the system (18) is chaotic.

For any two state vectors $x_{i}$ and $x_{j}$ of the Lorenz system, since chaotic attractor is bounded in a certain region, there exists a constant $\theta$ satisfying $\left\|x_{i k}\right\| \leq \theta$ and $\left\|x_{j k}\right\| \leq \theta$ for $k=1,2,3$. Then one gets the following

$$
\begin{aligned}
& \left\|f\left(x_{i}\right)-f\left(x_{j}\right)\right\|=\sqrt{\begin{array}{l}
\left(x_{j 1} x_{j 3}-x_{i 1} x_{i 3}\right)^{2} \\
+\left(x_{i 1} x_{i 2}-x_{j 1} x_{j 2}\right)^{2}
\end{array}} \\
& \leq 2 \theta\left\|x_{i}-x_{j}\right\|=\alpha\left\|x_{i}-x_{j}\right\|
\end{aligned}
$$

Then the Lorenz system satisfies the Hypothesis H1.

The complex dynamical network is assumed to contain 10 nodes and transmission noise is characterized by Guass stochastic noise with mean 0 and magnitude 0.1 . The other parameters of networks are $H=\left[\begin{array}{lll}1 & 0 & 0\end{array}\right]$, $\Gamma=\operatorname{diag}\left(\begin{array}{lll}1 & 1 & 1\end{array}\right)$, and $L=\left[\begin{array}{lll}1 & 1 & 1\end{array}\right]^{T}$. The initial values of networks are randomly evaluated in $[0,1]$.

A small world network coupled with states is considered. Using MATLAB LMI toolbox, one obtains $\gamma=2$

$$
P=\left[\begin{array}{cccc}
10.6167 & -4.8627 & -0.0003 & -0.1234 \\
-4.8627 & 2.2281 & -0.0008 & -0.0354 \\
-0.0003 & -0.0008 & 0.5995 & -0.0014 \\
-0.1234 & -0.0354 & -0.0014 & 20.6716
\end{array}\right]
$$

$$
K=\left[\begin{array}{c}
-16230.5230 \\
-35426.0276 \\
-56.9691 \\
-322.2331
\end{array}\right]
$$

The simulation results are shown in Figure 1. From Figure 1 , one can see that the error dynamics converge to zeros although there is transmission noise.

\section{Conclusion}

In this paper, we study the problem of state reconstructtion for a complex dynamical network under noisy circumstances. The state coupling network and the output coupling network are both considered. To attenuate noise in the transmission channel, integral observers are used and estimation errors with $H_{\infty}$ performance index are obtained. Some examples are given to demonstrate the effectiveness of the proposed scheme.

\section{REFERENCES}

[1] S. H. Strogatz, "Exploring Complex Networks," Nature, Vol. 410, No. 8, 2001, pp. 268-276. doi: $10.1038 / 35065725$

[2] E. De Silva and M. P. H. Stumpf, "Complex Networks and Simple Models in Biology," Journal of the Royal Society Interface, Vol. 2, No. 5, 2005, pp. 419-430. doi:10.1098/rsif.2005.0067

[3] T. A. S. Pardo, L. Antiqueira, M. D. G. Nunes, O. N. Oliveira and L. D. F. Costa, "Using Complex Networks for Language Processing: The Case of Summary Evaluation," 2006 International Conference on Communications, Circuits and Systems Proceedings, Guilin, 25-28 June 2006, pp. 2678-2682. doi:10.1109/ICCCAS.2006.285222

[4] S. J. Harrison and J. R. Dickinson, "A Metabolomic Analysis of Yeast Deletion Mutants Reveals Complex Networks of Control," Yeast, Vol. 20, No. S1, 2003, pp. S220- S220.

[5] M. Chen and Tsinghua Univ, "Chaos Synchronization in Complex Networks," IEEE Transactions on Circuits and Systems I: Regular Papers, Vol. 55, No. 5, 2008, pp. 13351346. doi:10.1109/TCSI.2008.916436

[6] X. F. Wang and G. Chen, "Synchronization in Scale Free Dynamical Networks: Robustness and Fragility," IEEE Transaction on Circuits Systems I: Fundamental Theory and Applications, Vol. 49, No. 1, 2002, pp. 54-62. doi:10.1109/81.974874

[7] X. Li and G. Chen, "Synchronization and Desynchronization of Complex Dynamical Networks: An Engineering Viewpoint," IEEE Transactions on Circuits and Systems I: Fundamental Theory and Applications, Vol. 50, No. 11, 2003, pp. 1381-1390. doi:10.1109/TCSI.2003.818611

[8] R. Carli, A. Chiuso, L. Schenato and S. Zampieri, "Optimal Synchronization for Networks of Noisy Double Integrators," IEEE Transactions on Automatic Control, Vol. 
56, No. 5, 2011, pp. 1146-1152. doi:10.1109/TAC.2011.2107051

[9] G. Wang, J. Cao and J. Lu, "Outer Synchronization between Two Nonidentical Networks with Circumstance Noise," Physica A: Statistical and Its Applications, Vol. 389, No. 7, 2010, pp. 1480-1488. doi:10.1016/i.physa.2009.12.014

[10] C.-X. Fan, G.-P. Jiang and F.-H. Jiang, "Synchronization between Two Complex Dynamical Networks Using Scalar Signals under Pinning Control," IEEE Transactions on Circuits and Systems I: Regular Papers, Vol. 57, No. 11, 2010, pp. 2991-2998. doi:10.1109/TCSI.2010.2048774

[11] X. Q. Wu, W. X. Zheng and J. Zhou, "Generalized Outer Synchronization between Complex Dynamical Networks," Chaos, Vol. 19, No. 1, 2009, p. 013109. doi:10.1063/1.3072787

[12] G.-P. Jiang, W. K.-S. Tang and G. Chen, "A State-Observer-Based Approach for Synchronization in Complex Dynamical Networks," IEEE Transactions on Circuits and Systems I: Regular Papers, Vol. 53, No. 12, 2006, pp. 2739-2745. doi:10.1109/TCSI.2006.883876

[13] R. M. Gutierrez-Ríos, J. A. Freyre-Gonzalez, O. Resendis, J. Collado-Vides, M. Saier and G. Gosset, "Identification of Regulatory Network Topological Units Coordinating the Genome-Wide Transcriptional Response to Glucose in Escherichia coli," BMC Microbiology, Vol. 7, No. 53, 2007. doi:10.1186/1471-2180-7-53

[14] W. K. S. Tang and L. Kocarev, "Identification and Monitoring of Biological Neural Network," IEEE International Symposium on Circuits and Systems, New Orleans, 27-30 May 2007, pp. 2646-2649. doi:10.1109/ISCAS.2007.377957

[15] J. Zhou and J.-A. Lu, "Topology Identification of Weighted Complex Dynamical Networks," Physica A: Statistical Mechanics and Its Applications, Vol. 386, No. 1, 2007, pp. 481-491. doi:10.1016/j.physa.2007.07.050

[16] H. Liu, G.-P. Jiang and C.-X. Fan, "State-Observer-Based Approach for Identification and Monitoring of Complex
Dynamical Networks," 2008 IEEE Asia Pacific Conference on Circuits and Systems (Apccas 2008), Macao, 30 November-3 December 2008, pp. 1212-1215. doi:10.1109/APCCAS.2008.4746244

[17] H. Liu, J. N. Lu and J. H. Lu, "Topology Identification of an Uncertain General Complex Dynamical Network," Proceedings of 2008 IEEE International Symposium on Circuits and Systems, Seattle, 18-21 May 2008, pp. 109-112. doi:10.1109/ISCAS.2008.4541366

[18] X. Wu, "Synchronization-Based Topology Identification of Weighted General Complex Dynamical Networks with Time-Varying Coupling Delay," Physica A: A-Statistical Mechanics and Its Applications, Vol. 387, No. 4, 2008, pp. 997-1008. doi:10.1016/j.physa.2007.10.030

[19] W. Guo, S. Chen and W. Sun, "Topology Identification of the Complex Networks with Non-Delayed and Delayed Coupling," Physics Letters A, Vol. 373, No. 41, 2009, pp. 3724-3729. doi:10.1016/j.physleta.2009.08.054

[20] H. Liu, J.-A. Lu, J. Lü and D. J. Hill, "Structure Identification of Uncertain General Complex Dynamical Networks with Time Delay," Automatica, Vol. 45, No. 8, 2009, pp. 1799-1807. doi:10.1016/j.automatica.2009.03.022

[21] G.-P Jiang, W. X. Zheng, W. K.-S. Tang and G. Chen, "Integral-Observer-Based Chaos Synchronization," IEEE Transactions on Circuits and Systems II: Express Briefs, Vol. 53, No. 2, 2006, pp. 110-114. doi:10.1109/TCSII.2005.857087

[22] Y. Liu, Z. Wang, J. Liang and X. Liu, "Synchronization and State Estimation for Discrete-Time Complex Networks with Distributed Delays," IEEE Transactions on Systems, Man and Cybernetics, Part B: Cybernetics, Vol. 38, No. 5, 2008, pp. 1314-1324. doi:10.1109/TSMCB.2008.925745

[23] K. K. Busawon and P. Kaboreb, "Disturbance Attenuation Using Proportional Integral Observers," International Journal of Control, Vol. 74, No. 6, 2001, pp. 618-627. doi: $10.1080 / 00207170010025249$ 